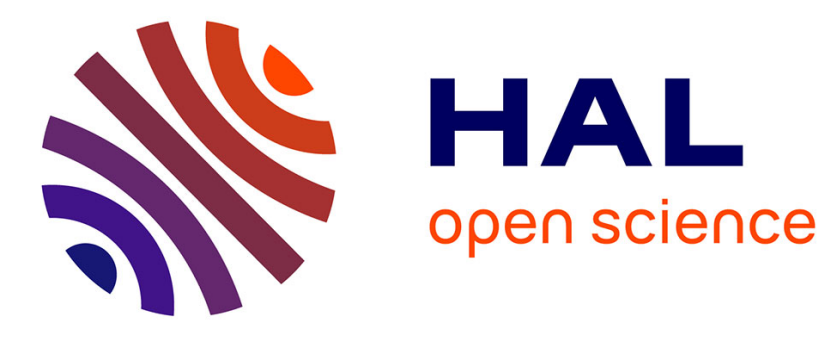

\title{
Nacre-like alumina composites based on heteroaggregation
}

Mariana Muňoz, Manuella Cerbelaud, Arnaud Videcoq, Hassan Saad, Alexandre Boulle, Sylvain Meille, Sylvain Deville, Fabrice Rossignol

\section{- To cite this version:}

Mariana Muňoz, Manuella Cerbelaud, Arnaud Videcoq, Hassan Saad, Alexandre Boulle, et al.. Nacrelike alumina composites based on heteroaggregation. Journal of the European Ceramic Society, 2020, 40 (15), pp.5773-5778. 10.1016/j.jeurceramsoc.2020.06.049 . hal-02938571

\section{HAL Id: hal-02938571 \\ https://hal.science/hal-02938571}

Submitted on 18 Nov 2020

HAL is a multi-disciplinary open access archive for the deposit and dissemination of scientific research documents, whether they are published or not. The documents may come from teaching and research institutions in France or abroad, or from public or private research centers.
L'archive ouverte pluridisciplinaire HAL, est destinée au dépôt et à la diffusion de documents scientifiques de niveau recherche, publiés ou non, émanant des établissements d'enseignement et de recherche français ou étrangers, des laboratoires publics ou privés. 


\title{
Nacre-like alumina composites based on heteroaggregation
}

\author{
Mariana Muñoz ${ }^{\mathrm{a}}$, Manuella Cerbelaud ${ }^{\mathrm{a}, *}$, Arnaud Videcoq ${ }^{\mathrm{a}}$, Hassan Saad ${ }^{\mathrm{b}}$, Alexandre \\ Boulle $^{\mathrm{a}}$, Sylvain Meille ${ }^{\mathrm{c}}$, Sylvain Deville ${ }^{\mathrm{b}, \mathrm{d}}$, Fabrice Rossignol $^{\mathrm{a}}$ \\ ${ }^{a}$ Univ. Limoges, CNRS, IRCER, UMR 7315, F-87000 Limoges, France \\ ${ }^{b}$ Laboratoire de Synthèse \& Fonctionnalisation des Céramiques, UMR3080 CNRS-Saint-Gobain, Saint \\ Gobain Research Provence, 84306 Cavaillon, France \\ ${ }^{c}$ Univ. Lyon, INSA-Lyon, MATEIS CNRS UMR5510, Villeurbanne, France \\ ${ }^{d}$ now with Univ. Lyon, Université Claude Bernard Lyon 1, CNRS, Institut Lumière Matière, 696222 \\ Villeurbanne, France
}

\begin{abstract}
High strength and high toughness are usually mutually exclusive in materials. Among all material classes, ceramics exhibit a high stiffness and strength, but they present a limited plastic deformation, which results in a moderate toughness. However, tough ceramics have been obtained using anisotropic particles organized in a 'brick and mortar' microstructure, inspired by the structure of the natural nacre. Here, we propose to build nacre-like ceramic composites from colloidal suspensions using heteroaggregation of particles. Two different shaping processes are used: direct settling of suspensions or freeze-granulation. After sintering, in both cases, the platelets alignment is very good, close to that of platelets in natural nacre, with a slightly better one noted for direct settling. Despite a better platelet alignment, the toughness is lower than in previous studies showing that further improvement of the interfacial phases present in the material must now be considered to reinforce its mechanical behavior.
\end{abstract}

Keywords: Nacre-like composite; Heteroaggregation; Platelet alignment; Settling; Freeze-granulation

\section{1. Introduction}

Nacre, found in several species of seashells, is a natural material composed of $95 \mathrm{vol} . \%$ of aragonite $\left(\mathrm{CaCO}_{3}\right)$ and $5 \mathrm{vol} . \%$ of organic materials (proteins), that exhibits a toughness at ${ }_{4}$ least three orders of magnitude higher than those of calcium carbonate $[1,2,3]$. The high

\footnotetext{
*Corresponding author

Email address: manuella.cerbelaud@unilim.fr; Tel: +33 (0)5 87502347 (Manuella Cerbelaud)
} 
toughness of nacre is conferred by its 'brick and mortar' hierarchical architecture composed of layers of inorganic and organic materials [2]. The alignment of inorganic bricks in nacre is remarkable, and has been shown to induce several toughening mechanisms [4]. In the last two decades, natural materials like nacre have been replicated to obtain brick and mortar composites with mechanical properties greater than those of their elementary constituents $[1,5,6,7]$.

To build brick and mortar materials, the control of platelet-shape particles alignment is critical [8]. To control this alignment, different techniques have been proposed [9] such as magnetic fields [10, 11], gravitational settling [12] or ice-templating [7, 13]. Most of these processes use colloidal suspensions which have to be well-dispersed. For that purpose, the use of organic binders or dispersants is common, which often imposes a debinding phase before sintering. An alternative, that avoids the introduction of organic compounds in the microstructure, is to take advantage of surface charges present at the surface of particles in aqueous suspensions. Previous studies have shown that the heteroaggregation of particles under the action of the electrostatic forces represents a promising method to control the arrangement of particles $[14,15,16]$.

Here, a nacre-like ceramic composite is elaborated without adding dispersant or carbonated additives unlike in the previous studies. Its mechanical properties are compared with the properties reported by Bouville et al. [7]. A similar composition is used: crystalline alumina platelets are used as bricks, and silica nanoparticles are incorporated to form a viscous phase during sintering acting as the mortar phase. Alumina nanoparticles are also added to create mechanical anchorage between platelets and reinforce the materials. After consolidation by sintering the desired structure of the composite is thus composed of layers of micron-size platelets separated by a silica vitreous phase and alumina bridges. In order to avoid carbon in the composition to better control the composite color, the vitreous phase formed in this study does not contain calcium carbonate and thus is different from that reported in [7].

Here, we show that such nacre-like ceramic composites can be obtained without addition of organic additives using simple shaping methods. Brownian dynamics simulations and experiments have indeed shown that using the heteroaggregation between the alumina platelets and the silica nanoparticles in aqueous suspensions yields at the same time a compact arrangement of the platelets and a good spatial distribution of the silica in between them [16]. 
To shape the composites, two simple methods are used. The first one consists in the natural settling of suspensions and the second in freeze-granulation and lyophilisation. A thermal treatment is then applied to consolidate green samples obtained from both shaping methods. Observations of sintered microstructures and measurements of platelets alignment are performed. Finally, mechanical properties of elaborated composites are determined to establish a relationship between the microstructure and the mechanical performance.

\section{Methodology}

The alumina platelets $\left(\mathrm{Al}_{2} \mathrm{O}_{3}\right)$ used in this study are supplied by Merck, Germany (sapphire alumina RonaFlair@White Sapphire, $\mathrm{d}_{50} \sim 7 \mu \mathrm{m}$, purity $\geq 99 \%$ ). Silica nanoparticles $\left(\mathrm{SiO}_{2}\right)$ with a mean particle diameter of $25 \mathrm{~nm}$ are obtained from a commercial aqueous suspension of Ludox@TM50 supplied by Grace Davidson, United States. High purity $\alpha$-alumina nanoparticles of about $100 \mathrm{~nm}\left(\alpha \mathrm{Al}_{2} \mathrm{O}_{3}\right)$, used as reinforcement, are from Taimei Chemical Company, Japan (TM-DAR, Taimicron, purity $>99.99 \%$ ).

The zeta potential of particles as a function of $\mathrm{pH}$ is measured using Acoustosizer IIs from Colloidal Dynamics. For the measurements, aqueous suspensions are prepared with a solid loading of $1 \mathrm{wt} . \%$ in osmosed water and $\mathrm{pH}$ is adjusted using $\mathrm{HCl}$ and $\mathrm{NaOH}$.

The mixed suspensions, used for shaping the nacre-like ceramic composites, are prepared by adding the various constituents in osmosed water and are deagglomerated by an ultrasonic treatment $(300 \mathrm{~W}, 30 \mathrm{~s}$, pulse on $3 \mathrm{~s}$, pulse off $1 \mathrm{~s})$. The volume ratio between the various components $\mathrm{Al}_{2} \mathrm{O}_{3} / \alpha \mathrm{Al}_{2} \mathrm{O}_{3} / \mathrm{SiO}_{2}$ is fixed at $94.6 \% / 3.6 \% / 1.8 \%$.

Sedimentation tests are performed for suspensions prepared with a solid loading of 3vol.\%. For that, suspensions are prepared and introduced in closed tubes, and allowed to settle. When all suspensions are settled, the sediment heights are measured. The suspensions are also characterized by environmental scanning electron microscopy (SEM) on a Quanta FEG 450 from FEI. Droplets of suspensions are deposited directly on the specimen holder. Drying is performed in real time during the observations in the microscope.

In this study, two methods are applied to shape the composites: - The first method consists in obtaining an alumina-based green part in the shape of a pellet (diameter $25 \mathrm{~mm}$ with a thickness comprised between 5 and $13 \mathrm{~mm}$ ) by settling the suspensions directly in a rubber silicone mold. The water excess is eliminated after 24 hours of 
settling and, before demolding, samples are left for natural drying during 24 hours.

- The second method consists in obtaining powders from the elaborated suspensions, using freeze granulation and freeze-drying. Freeze granulation is performed with a freeze granulator LS-2 from PowderPro AB. Air is used as the atomization gas. The atomization conditions are: air relative pressure of 0.3 bar and suspension flow rate of $33.5 \mathrm{~mL} / \mathrm{min}$. The rotation speed of the magnetic stirrer in the liquid nitrogen is set to $370 \mathrm{rpm}$. After granulation, the frozen ganules are freeze-dried in a Christ Beta-1-8-LDplus under a final vacuum of $10^{-3} \mathrm{mbar}$.

For both shaping methods, the same thermal treatment stage is used. Pellets or powders are sintered using Field Assisted Sintering (FAST, Dr. Sinter Model 825, SUGA, Japan), deriving benefit of the high heating and cooling rates provided (heating rate: $100^{\circ} \mathrm{C} / \mathrm{min}$ and cooling rate: $50^{\circ} \mathrm{C} / \mathrm{min}$ ). Graphite dies are used for all temperature profiles and a maximum temperature of $1400{ }^{\circ} \mathrm{C}$ and a pressure of $100 \mathrm{MPa}$ are reached. A dwell of $5 \mathrm{~min}$ in these conditions is performed before cooling.

For both upstream shaping methods, dried samples are characterized by SEM (LEO $1530 \mathrm{vp)}$ before and after thermal treatment to observe the distribution of particles and the alignment alumina platelets in the material.

The alignment of platelets in the sintered samples is characterized by X-ray diffraction (XRD), using a Bruker D8 "Discover" diffractometer equipped with a copper target ( $\mathrm{Cu} K_{\alpha 1}$ radiation). A parabolic mirror coupled with a two-reflection Ge (220) monochromator provides a monochromatic and quasi-parallel X-ray beam. Diffracted X-ray are collected with a linear position sensitive detector covering a $2^{\circ}$ angular range with $0.01^{\circ}$ resolution. The sintered samples are positioned on a 3-circle goniometer allowing to precisely align the sample surface with respect to the incident beam. XRD pole figures are recorded from the (0012) planes of the corundum structure of $\mathrm{Al}_{2} \mathrm{O}_{3}$. A pole figure displays the intensity diffracted from a selected set of planes in the hemisphere above the sample and is commonly represented in a polar plot (stereographic projection) where the angle along the circumference correspond to the rotation about the surface normal ( $\Phi$ angle), and the radius is the inclination angle of the crystallographic planes ( $\Psi$ angle). In the present case $\Phi$ are scanned from $-180^{\circ}$ to $180^{\circ}$ with $5^{\circ}$ steps, whereas $\Psi$ has been scanned from to 0 to $80^{\circ}$ with $1^{\circ}$ steps.

Finally, to measure the mechanical properties of the obtained materials, Single Edge Notched Beam (SENB) and bending tests are carried out. Rectangular beams of dimensions $B=2 \mathrm{~mm}$ 
(thickness), $W=2.5 \mathrm{~mm}$ (width) and $L=20 \mathrm{~mm}$ (length) are cut from the sintered pellets. The material is solicited perpendicularly to the platelets direction ie along $W$. Three points bending (3PB) configurations are used following the ASTM E1820-E01 standard [17] to evaluate both the flexural strength of the material and its fracture toughness in terms of crack-resistance curve (R-curve). All tests are carried out using a Shimadzu AGS-X machine equipped with a $10 \mathrm{kN}$ load cell and using a Linear Variable Differential Transformer (LVDT) with a precision of $1 \mu \mathrm{m}$ to measure the beam deflection. For each set-up, 4 samples are tested.

The flexural strength of the material is determined from un-notched and chamfered specimens. In another hand, SENB specimens are first notched using a $300 \mu \mathrm{m}$ diamond blade. The bottom of the pre-notch is sharpened using a razor blade with $1 \mu \mathrm{m}$ diamond paste. Final notches with radius of curvature between 15 and $20 \mu \mathrm{m}$ are obtained to favour stable crack propagation for the R-curves measurements. In both configurations, the specimen are loaded monotonically at a constant displacement rate of $1 \mu \mathrm{m} \cdot \mathrm{s}^{-1}$ with a support $\operatorname{span}(S)$ of $16 \mathrm{~mm}$. The compliance method $\left(C=\frac{d}{F}\right)$ is used to determine the beginning of the crack propagation and to determine the projected crack length during SENB tests using the following recursive equation :

$$
a_{n}=a_{n-1}+\frac{W-a_{n-1}}{2} \frac{C_{n}-C_{n-1}}{C_{n}}
$$

with $a$ and $C$ the crack length and the compliance respectively, calculated at the $n$ and the $n-1$ steps. Non-linear elastic fracture mechanisms analysis is used to determine the crack-resistance curves of the material ( $R$-curves). $R$-curves are measured in terms of the $J$-integral as a function of the crack extension in order to capture both intrinsic and extrinsic mechanisms acting as crack-arresters. The $J$-integral is computed taking into account an elastic $\left(J_{e l}\right)$ and a plastic $\left(J_{p l}\right)$ contribution : $J=J_{e l}+J_{p l}$. The elastic contribution $J_{e l}$ is calculated using classic linear-elastic fracture mechanics relations : $J_{e l}=\frac{K^{2}}{E^{\prime}}$ where $E^{\prime}=E$ (Young's modulus) in plane stress and $E^{\prime}=\frac{E}{1-\nu}$ in plane strain ( $\nu$ is the Poisson's ratio). The plastic component $J_{p l}$ is defined as $J_{p l}=\frac{1.9 A_{p l}}{B b}$ where $A_{p l}$ is the plastic area under the $F-d$ curve and $b$ the uncracked ligament size. The standard mode I $J-K$ equivalence (eq. 2) permits to back-calculate, from the geometric mean of the local stress intensity factor $J$, an equivalent stress intensity factor $K_{J}$ :

$$
K_{J}=\left(J E^{\prime}\right)^{1 / 2}
$$


Toughness parameters such as crack initiation toughness $\left(\mathrm{K}_{I 0}\right)$ and fracture toughness $\left(K_{I C}\right)$ are directly determined from the R-curves. According to the ASTM criterion, the maximum crack extension capacity for a specimen is given by $\Delta a_{\max }=0.25 b_{0}$ where $b_{0}=W-a_{0}$ is the initial uncracked ligament. For $R$-curves calculation, the considered values for Young Modulus and Poisson ratio are respectively $E=368 \mathrm{GPa}$ and $\nu=0.24$ since we work with a system quasi-fully composed of alumina.

\section{Results}

\subsection{Heteroaggregation}

The first step in this study is to characterize the particles surface charges in aqueous suspensions. For each type of particle, zeta potential measurements as a function of $\mathrm{pH}$ are performed (Fig. 1a).

The natural pH of both alumina platelets and nanoparticles suspensions is around 6.5. An isoelectric point around 9 is found for both suspensions: for $\mathrm{pH}$ less than 9 , both $\mathrm{Al}_{2} \mathrm{O}_{3}$ platelets and nanoparticles are mainly positively charged and for $\mathrm{pH}$ greater than 9, they are negatively charged. For silica particles, the natural $\mathrm{pH}$ is around 9, which can be explained by the alkaline medium used to stabilize the commercial Ludox TM50 suspension. The zeta potential of silica is negative on the $2-10 \mathrm{pH}$ range. The goal of this study is to take advantage from the heteroaggregation between the silica nanoparticles and the alumina platelets as proposed in Ref. [16]. The natural pH of the three-component suspensions being around 7.6, a pH adjustment is not needed. At pH 7.6 (Fig. 1a), both alumina platelets and silica nanoparticles are oppositely charged, and therefore prone to heteroaggregation.

It has been shown [16], that the heteroaggregation of silica nanoparticles and alumina platelets can help disaggregating the suspension. In this study, a third component is used, the $\alpha \mathrm{Al}_{2} \mathrm{O}_{3}$ nanoparticles. To observe the effect of this third component on the suspension behavior, settling tests are performed. Figure $1 \mathrm{~b}$ shows the results obtained after 5 days with a suspension composed of alumina platelets only, with a suspension containing the alumina platelets and the $\alpha$-alumina nanoparticles and with a suspension containing the three components. It is observed that even with the addition of $\alpha \mathrm{Al}_{2} \mathrm{O}_{3}$ nanoparticles, the suspension remains stable. The sediment height of the three-component suspension is indeed lower than the two others. 

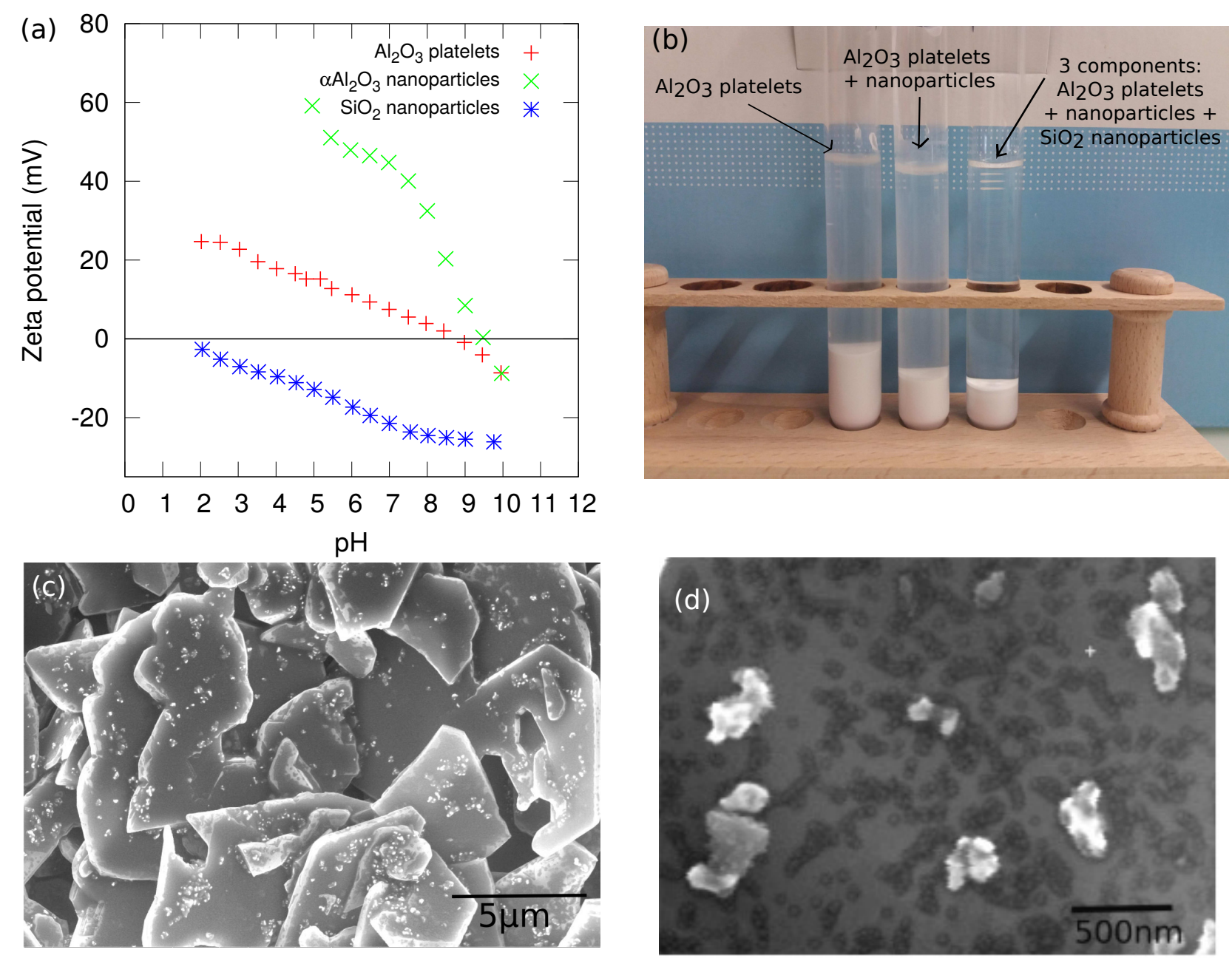

Figure 1: (a) Evolution of the zeta potential as a function of $\mathrm{pH}$ for the different components used in the nacre-like composite. Results of alumina platelets and silica nanoparticles are extracted from reference [16]. (b) Pictures of the closed tubes used in the sedimentation tests after 5 days. From left to right: a suspension of $\mathrm{Al}_{2} \mathrm{O}_{3}$ only, a binary suspension composed of $\mathrm{Al}_{2} \mathrm{O}_{3}$ and $\alpha \mathrm{Al}_{2} \mathrm{O}_{3}$ and a suspension with the three components $\left(\mathrm{Al}_{2} \mathrm{O}_{3}, \alpha \mathrm{Al}_{2} \mathrm{O}_{3}\right.$, and $\left.\mathrm{SiO}_{2}\right)$. (c) and (d) environmental SEM pictures of the three-component suspension (Pressure 670 Pa, HV 10kV, Humidity: 94.5\%). C)2020 M. Cerbelaud et al. (10.6084/m9.figshare.12018501) CC BY 4.0 license https://creativecommons.org/licenses/by/4.0/. 
The three-component suspension is observed by environmental SEM. Figures 1d shows silica nanoparticles on the alumina platelet faces. Moreover, aggregates composed of alumina and silica nanoparticles are also present on the platelets. These observations are consistent with the aforementioned charges of particles. Silica is negatively charged, therefore it is absorbed onto $\mathrm{Al}_{2} \mathrm{O}_{3}$ platelets and $\alpha \mathrm{Al}_{2} \mathrm{O}_{3}$ nanoparticles surfaces which are themselves positively charged. This observed arrangement at this early stage of the process is promising to obtain the desired microstructure in the composites. Silica nanoparticles and $\alpha \mathrm{Al}_{2} \mathrm{O}_{3}$ nanoparticles are both well spread onto the alumina platelet surfaces.

The three-component suspensions are then used to shape nacre-like ceramic composites. First, we use sedimentation to shape pellets. After drying, some pellets are broken in half and the rupture surface is observed by SEM. Figure 2a shows that in the foreground some of the platelets are disorganized due to the fracture of the material, however in the background a preferential orientation of alumina platelets in the pellets is observed. At this stage, a sedimentation by gravity yields a good alignment of platelets, which form layers in the samples. In between those layers, $\alpha \mathrm{Al}_{2} \mathrm{O}_{3}$ nanoparticles are dispersed forming potential anchorage after consolidation by sintering. The dispersion of silica nanoparticles on platelets faces (Fig. 2b), already observed in the suspensions (Figure 1d), is maintained in the green sample.

Composites are also made by the freeze-granulation method. Because of the low solid loading of the suspensions used for granulation, the granules are not self-supporting and only aggregated powder is obtained at the end. Figures $2 \mathrm{c}$ and $2 \mathrm{~d}$ show the SEM pictures of the powder obtained after the freeze-granulation. An homogeneous dispersion of the different components is observed. Both $\alpha \mathrm{Al}_{2} \mathrm{O}_{3}$ and silica nanoparticles are well-dispersed on the platelets surface.

\subsection{Microstructural characterization of the sintered samples}

The green samples are then sintered by FAST. After the thermal treatment, an important linear shrinkage $(\sim 70 \%)$ is observed in the sedimentation direction for the pellets obtained by sedimentation. Archimede's Law of Buoyancy gives an apparent density of $3.93 \mathrm{~g} . \mathrm{cm}^{-3}$ and an open porosity of $0.36 \%$ for the sample obtained by the sedimentation route and an apparent density of $3.85 \mathrm{~g} . \mathrm{cm}^{-3}$ and an open porosity of $0.48 \%$ for the sample obtained by a freeze-granulation route. After fracture, a cross section of both samples is mirror polished and 

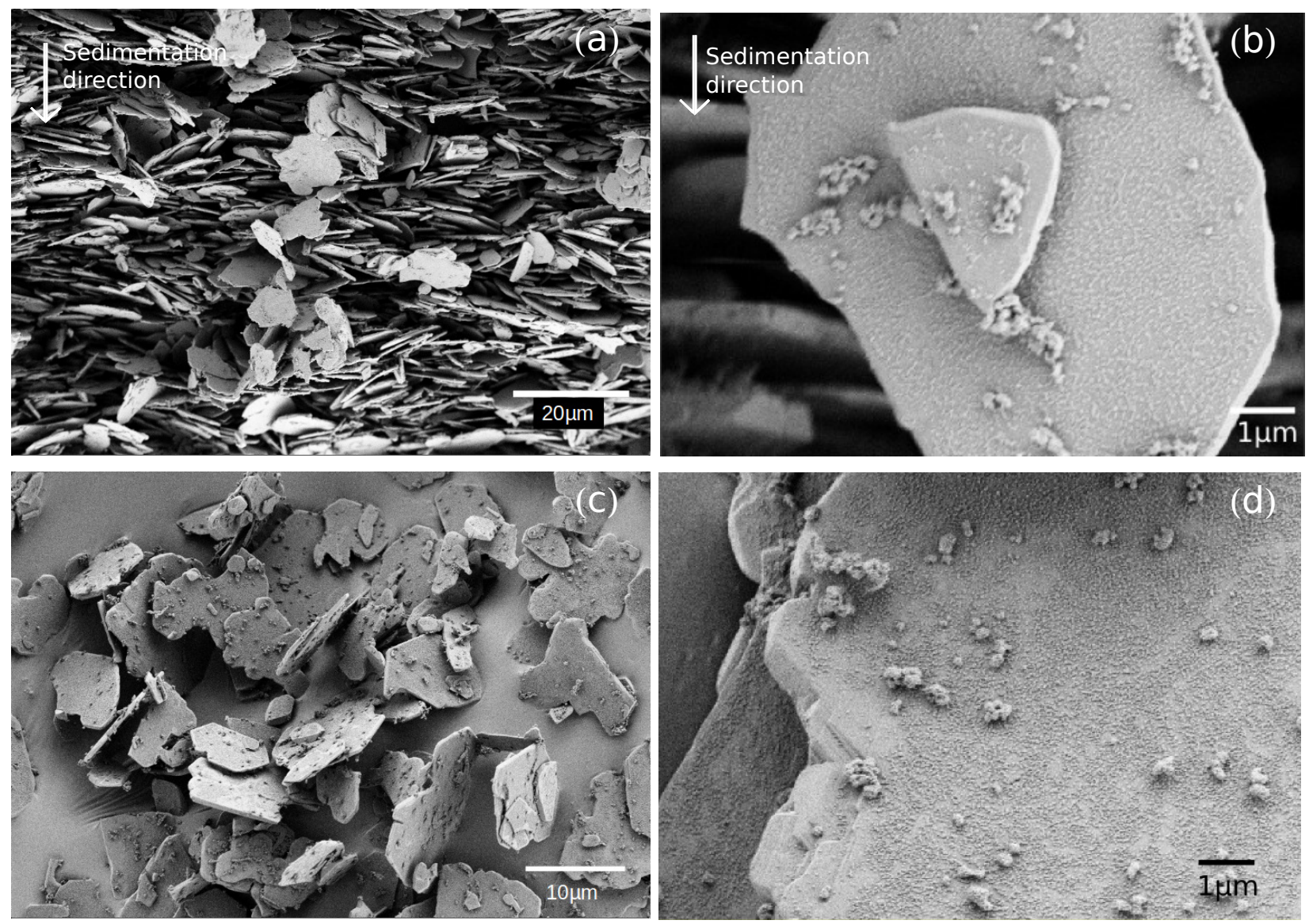

Figure 2: (a) and (b) SEM pictures of a fracture surface of a dried pellet obtained by sedimentation. (c) and (d) SEM pictures of the powder obtained by freeze-granulation. (C)2020 M. Cerbelaud et al. (10.6084/m9.figshare.12018501) CC BY 4.0 license https://creativecommons.org/licenses/by/4.0/. 


\begin{tabular}{c|c|c|} 
& Average strength (MPa) & Standard deviation (MPa) \\
\hline Sedimentation & 454 & 20 \\
\hline $\begin{array}{c}\text { Freeze granulation } \\
\text { and freeze-drying }\end{array}$ & 338 & 46 \\
\hline
\end{tabular}

Table 1: Averaged flexural strength of samples from both shaping methods and post sintered by FAST (averaged over 4 samples).

observed by SEM (Fig. 3a and 3b). Alumina platelets are easily recognized on the pictures because of the contrast difference. A good alignment of platelets is observed in both cases, with a slightly higher disorder in the freeze-granulated samples. The (0012) XRD pole figures of both types of samples are shown in Figures 3c and 3d. An estimation of average platelet disorientation can be obtained by computing the root-mean-squared disorientation obtained from a Gaussian fit of each $\Psi$ scan building up the pole figure. With this approach we find an average disorientation of platelets of $7.5 \pm 0.4^{\circ}$ for the sample obtained by sedimentation and $8.2 \pm 0.2^{\circ}$ for the samples obtained by freeze-granulation. The disorientation of the platelets is very low, and comparable to that of the natural nacre which is about $5^{\circ}[4]$. The platelet alignment is better than that obtained by ice templating and reported by Bouville et al. [7] $\left(15^{\circ}\right)$.

\subsection{Mechanical properties}

The strength of the samples is measured by three points bending. Table 1 shows the average strength of composites obtained from both methods.

The flexural strength values obtained are equivalent to those reported for crystalline alumina $[18,19]$ and comparable to the results of Bouville et al. (470 MPa) [7].

The flexural strength values are a slightly higher for samples shaped by sedimentation. Because the composition and the tests conditions are the same for both approaches, this result might be related to the lower porosity and the better alignment of platelets obtained by sedimentation. To measure the toughness of the elaborated composites, SENB tests are carried out, and results are compared to those obtained by Bouville et al. [7]. The stressstrain curves are treated to obtain the fracture toughness of composites. Four specimens for each approach are tested to assess reproducibility. The initiation fracture toughness $\left(\mathrm{K}_{1 c}\right)$ obtained for the composite elaborated from the sedimentation route is $3.8 \mathrm{MPa} \cdot \mathrm{m}^{1 / 2}$, and 

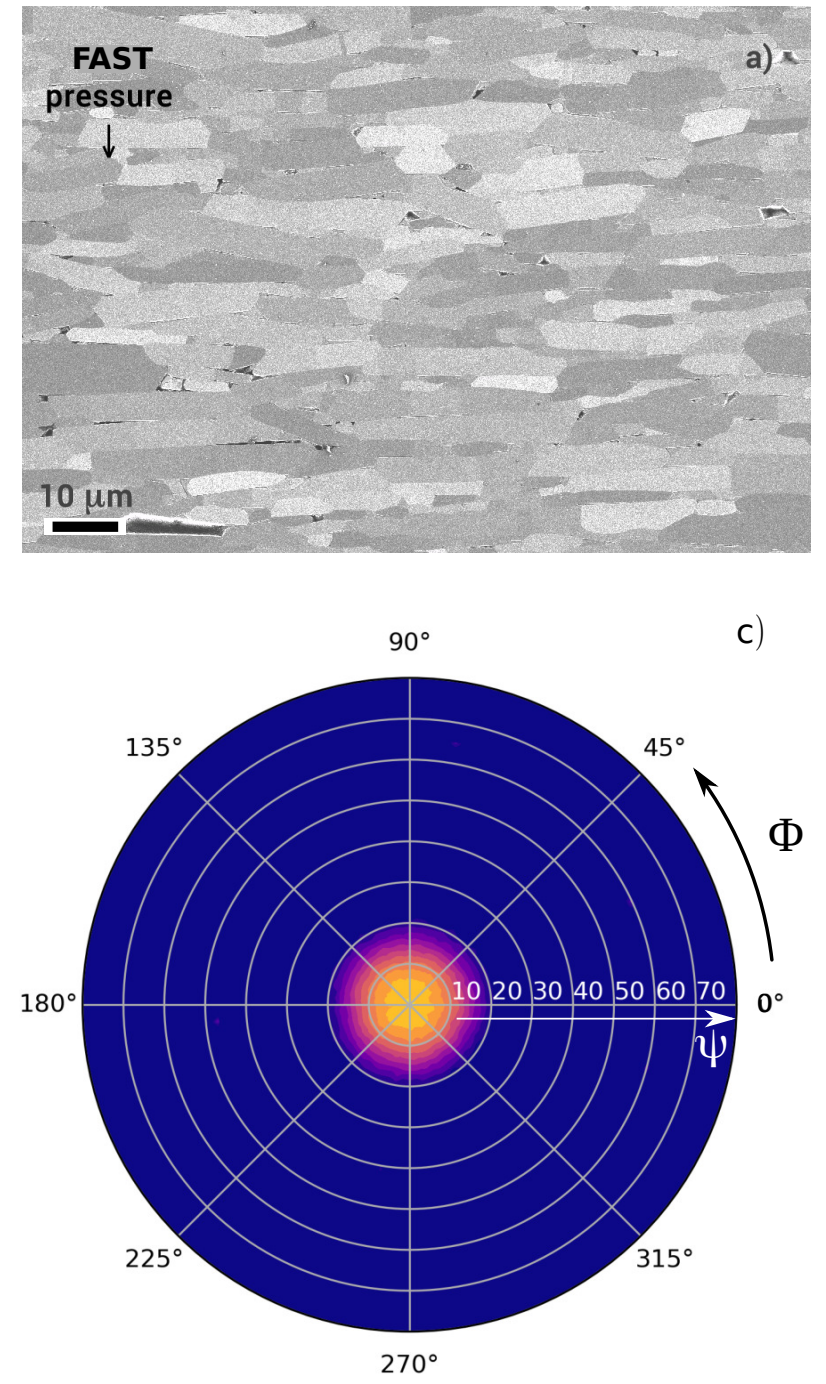
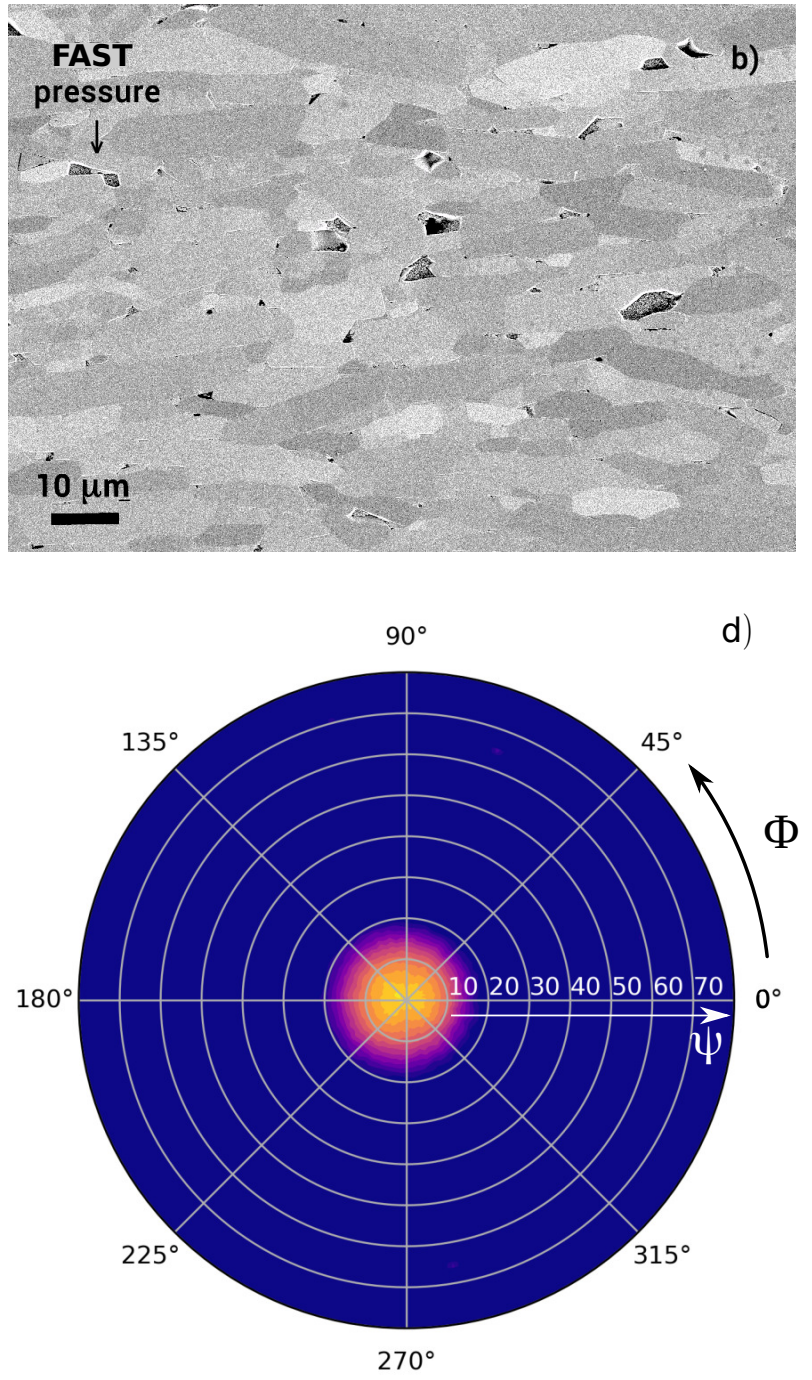

Figure 3: SEM pictures of polished sections of sintered samples and corresponding XRD pole figures used to determine the platelets disorientation: (a) and (c) samples obtained by sedimentation; (b) and (d) samples obtained by freeze granulation. (C)2020 M. Cerbelaud et al. (10.6084/m9.figshare.12018501) CC BY 4.0 license https://creativecommons.org/licenses/by/4.0/. 

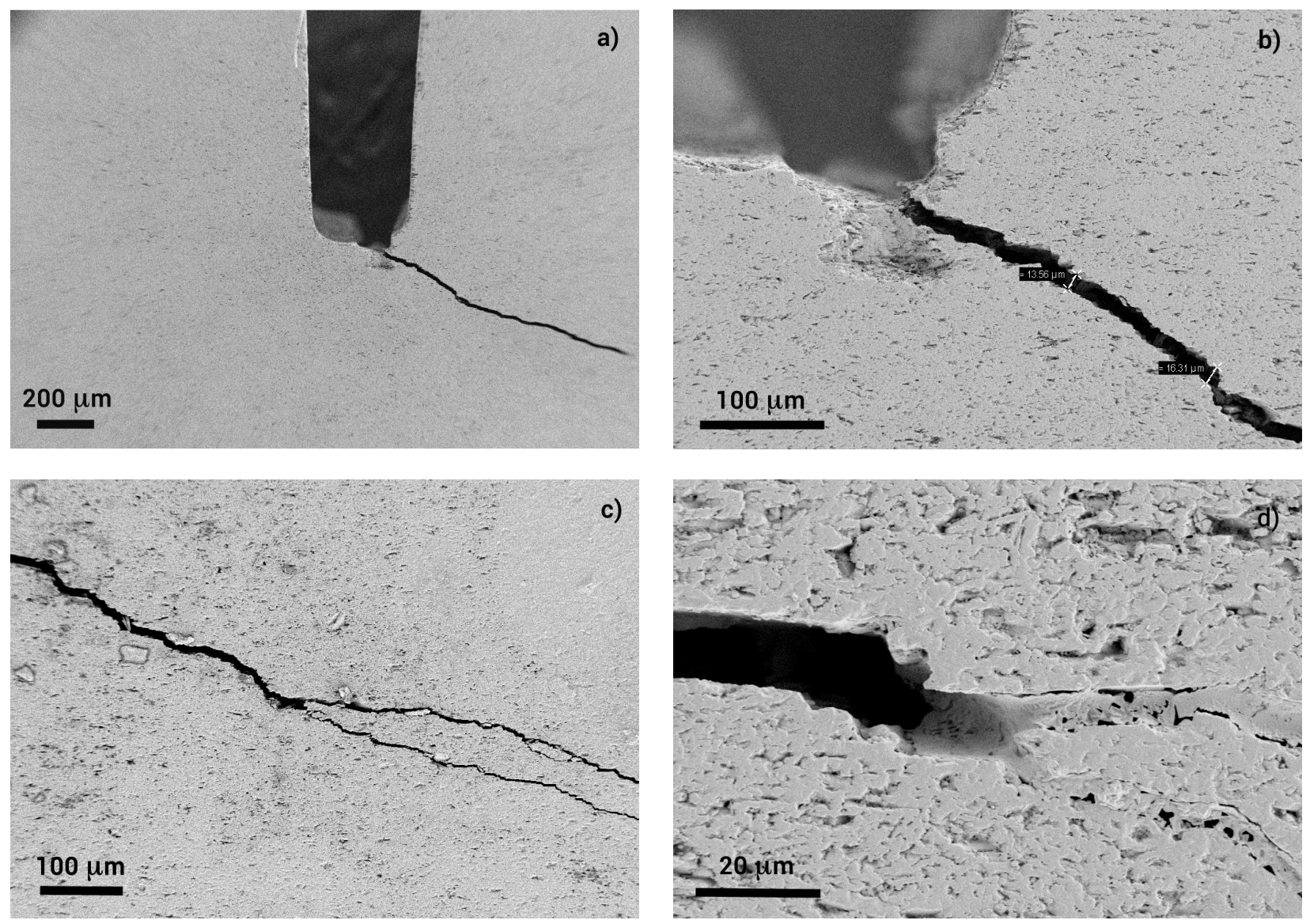

Figure 4: SEM pictures of cracks propagation observed on the samples used for the SENB tests. (a) and (b): Area where crack is initiated: deflection; (c) and (d) crack after propagation: multiple cracks and crack bridging. (C)2020 M. Cerbelaud et al. (10.6084/m9.figshare.12018501) CC BY 4.0 license https://creativecommons.org/licenses/by/4.0/.

that of the composite obtained from freeze-granulation is $3.6 \mathrm{MPa} \cdot \mathrm{m}^{1 / 2}$. These values are equivalent to the reference values of fracture toughness for alumina $\left(3.5 \mathrm{MPa} . \mathrm{m}^{1 / 2}\right)$ but are lower than the result for the nacre-like composite from Bouville et al. $\left(6.1 \mathrm{MPa} \cdot \mathrm{m}^{1 / 2}\right)$ [7]. For both composites crack propagation shows strong deviation at the interface between platelets, multiple cracks are observed as well as some crack bridging (Fig. 4). These toughening mechanisms, crack deflection and crack bridging, are similar to those observed in natural materials such nacre or bonds, presenting a high toughness [20,21, 22, 23]. They lead to the increase of fracture resistance with the propagation of cracks (i.e. R-curves). The Rcurves for both composites are plotted in Figure 5. The measurement is done according to ASTM E-1820 [17] and is considered as valid until a maximum crack length determined by $\Delta a_{\max }=0.25 \mathrm{~b}_{0}$, where $\mathrm{b}$ is the thickness of the specimen ligament. In our case, this 

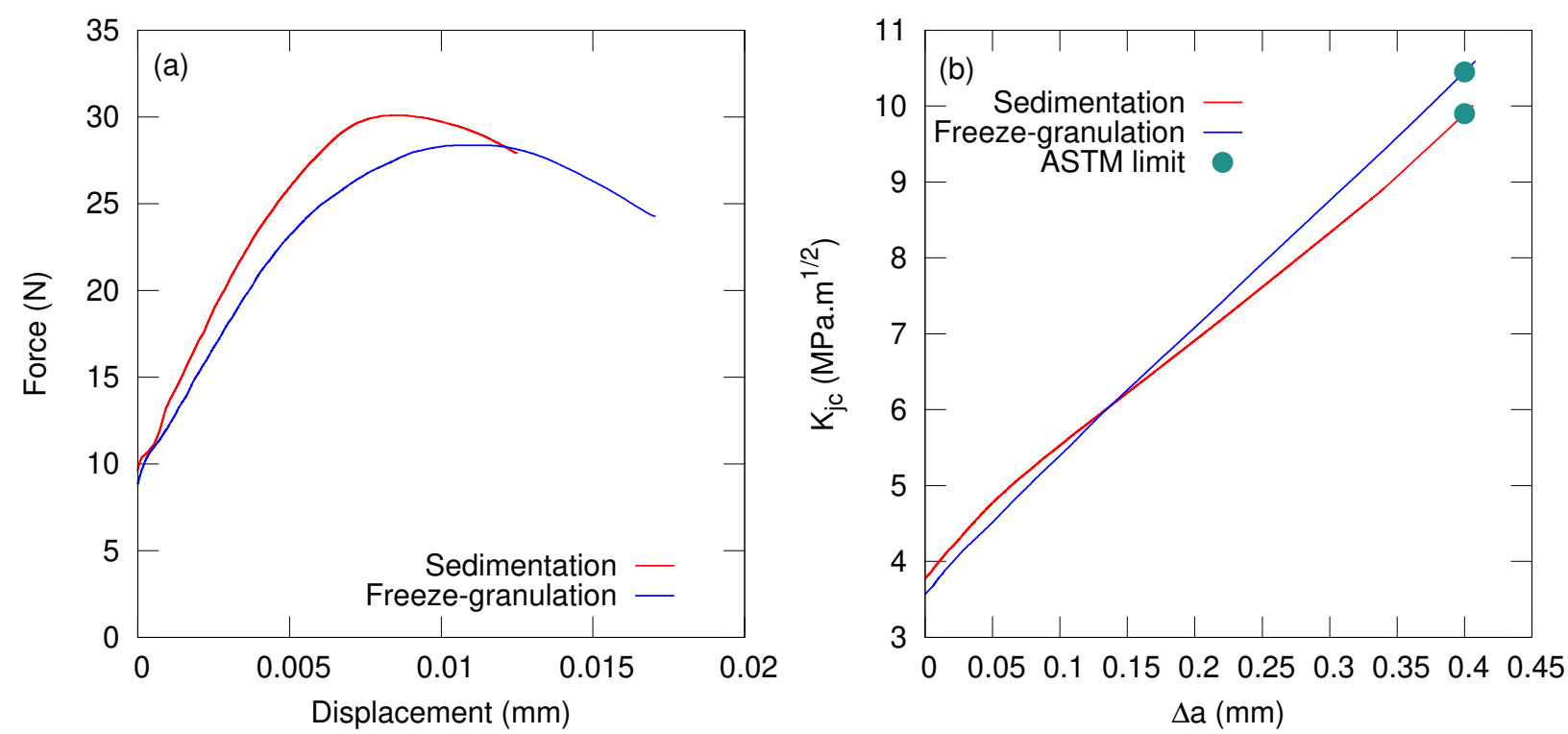

Figure 5: (a) Evolution of the force as a function of the displacement for the three points bending tests on samples with pre-notches obtained by sedimentation and by freeze-granulation routes and (b) corresponding R-curves : Evolution of the fracture toughness $\mathrm{K}_{j c}$ calculated from the J-integral as a function of crack extension $\Delta$ a. (C)2020 M. Cerbelaud et al. (10.6084/m9.figshare.12018501) CC BY 4.0 license https://creativecommons.org/licenses/by/4.0/.

maximum crack length is $0.4 \mathrm{~mm}$. The maximum toughness obtained is $10.1 \mathrm{MPa} \cdot \mathrm{m}^{1 / 2}$ and 10.6 MPa.m $\mathrm{m}^{1 / 2}$ for composites from the sedimentation route and the freeze-granulation route, respectively. These values are lower than the $17 \mathrm{MPa} \cdot \mathrm{m}^{1 / 2}$, obtained for the nacre-like alumina composite of Bouville et al. [7]. According to the observations, cracks propagate in the vitreous phase, however it can be noticed that the composition of the vitreous phase in these composites is slightly different from the one of the composites of Bouville et al. [7] which is obtained from a mixture of silica and calcium carbonate. This difference of composition could be at the origin of the lower mechanical performances reported here. Moreover, a greater grain growth is obtained in our samples probably due to the chemical composition of the intergranular phase, which can also be detrimental to the mechanical properties.

\section{Conclusion}

Nacre-like ceramic composites are obtained from heteroaggregated suspensions of alumina and silica particles by two methods: sedimentation and freeze-granulation. For both methods, 
a good distribution of the components is obtained, as well as a very good alignment of alumina platelets in the sintered samples (disorientation of $7.5^{\circ}$ and of $8.2^{\circ}$ for the sedimentation and freeze-granulation routes, respectively). The study of mechanical properties of the ceramic composites shows typical toughening mechanisms of brick and mortar composite materials, with crack deflection, multiple cracks, and crack bridging. The heteroaggregation between the silica nanoparticles and the alumina platelets is therefore a good alternative to obtain nacre-like ceramic composites without polymeric dispersant. In comparison, the composite obtained by the sedimentation route have slightly better flexural strength, which is attributed to a better platelet alignment.

Despite a better alignment of platelets, the mechanical properties are however lower than those obtained previously by freeze-casting [7]. This could be attributed to the difference of the vitreous phase composition, which appears to be a key point to improve the mechanical properties of nacre-like ceramic composites.

\section{Acknowledgements}

The authors want to acknowledge Saint-Gobain Research Provence for the access to samples preparation and mechanical tests.

\section{Funding}

This work is supported by an institutional grant of the French National Agency of Research (ANR-16-CE08-0006-0A BICUIT project).

[1] E. Munch, M. Launey, D. Alsem, E. Saiz, A. Tomsia, R. Ritchie, Tough, bio-inspired hybrid materials, Science 322 (2008) 1516-1520.

[2] R. Richtie, The conflicts between strength and toughness, Nat. Mater. 10 (2011) 817822.

[3] F. Barthelat, H. Tang, P. Zavattieri, C.-M. Li, H. Espinosa, On the mechanics of motherof-pearl: A key feature in the material hierarchical structure, J. Mech. Phys. Solids 55 (2007) 306-337.

[4] S.S.Xie, O. Vasylkiv, A.I.Y.Tok, Highly ordered nano-scale structure in nacre of greenlipped mussel perna canaliculus, CrystEngComm 18 (2016) 7501-7505. 
[5] L. Bonderer, A. Studart, L. Gauckler, Bioinspired design and assembly of platelet reinforced polymer films, Science 319 (2008) 1069-1073.

[6] Z. Tang, N. Kotov, S. Magonov, B. Ozturk, Nanostructured artificial nacre, Nat. Mater. 2 (2003) 413-418.

[7] F. Bouville, E. Maire, S. Meille, B. V. de Moortèle, A. Stevenson, S. Deville, Strong, tough and stiff bioinspired ceramics from brittle constituents, Nat. Mater. 13 (2014) $508-514$.

[8] A. Studart, Towards high-performance bioinspired composites, Adv. Mater. 24 (2012) $5024-5044$

[9] N. Yaraghi, D. Kisailus, Biomimetic structural materials: Inspiration from design and assembly, Annu. Rev. Phys. Chem. 69 (2018) 23-57.

[10] F. Bargardi, H. L. Ferrand, R. Libanori, A. Studart, Bio-inspired self-shaping ceramics, Nat. Commun. 7 (2016) 13912.

[11] R. Erb, J. Segmehl, M. Charilaou, J. Löffler, A. Studart, Non-linear alignment dynamics in suspensions of platelets under rotating magnetic fields, Soft Matter 8 (2012) 76047609 .

[12] S. Behr, U. Vainio, M. Müller, A. Schreyer, G. A. Schneider, Large-scale parallel alignment of platelet-shaped particles through gravitational sedimentation, Sci. Rep. 5 (2015) 9984.

[13] H. Bai, Y. Chen, B. Delattre, A. Tomsia, R. Ritchie, Bioinspired large-scale aligned porous materials assembled with dual temperature gradients, Sci. Adv. 1 (2015) e1500849.

[14] M. Cerbelaud, A. Videcoq, P. Abélard, C. Pagnoux, F. Rossignol, R. Ferrando, Heteroaggregation between $\mathrm{Al}_{2} \mathrm{O}_{3}$ submicrometer particles and $\mathrm{SiO}_{2}$ nanoparticles : Experiments and simulation, Langmuir 24 (2008) 3001-3008.

[15] M. Cerbelaud, A. Videcoq, P. Abélard, C. Pagnoux, R. Ferrando, Self-assembly of oppositely charged particles in dilute ceramic suspensions: predictive role of simulations, Soft Matter 6 (2010) 370-382. 
[16] M. Cerbelaud, M. Muñoz, F. Rossignol, A. Videcoq, Self-organization of large alumina platelets and silica nanoparticles by heteroaggregation and sedimentation: toward an alternative shaping of nacre-like ceramic composites, Langmuir DOI: 10.1021/acs.langmuir.0c00170.

[17] ASTM E1820-01, standard test method for measurement of fracture toughness, Standard, ASTM International, West Conshohocken, PA (2001). URL www. astm.org

[18] L. Ćurković, A. Bakić, J. Kodvanj, T. Haramina, Flexural strength of alumina ceramics: Weibull analysis, Trans. FAMENA 34 (2010) 13-19.

[19] S. Usami, H. Kimoto, I. Takahashi, S. Shida, Strength of ceramic materials containing small flaws, Eng. Fract. Mech. 23 (1986) 745-761.

[20] H. Peterlik, P. Roschger, K. Klaushofer, P. Fratzl, From brittle to ductile fracture of bone, Nat. Mater. 5 (2006) 52-55.

[21] K. Koester, J. AgerIII, R. O. Ritchie, The true toughness of human cortical bone measured with realistically short cracks, Nat. Mater. 7 (2008) 672-677.

[22] K. Okumura, P.-G. de Gennes, Why is nacre strong? elastic theory and fracture mechanics for biocomposites with stratified structures, Eur. Phys. J. E 4 (2001) 124-127.

[23] K. Faber, A. Evans, Crack deflection processes-II. experiment, Acta Metall. 4 (1983) $577-584$. 